\title{
Confirmatory cut point has limited ability to make accurate classifications in immunogenicity assays
}

\author{
Robert J Kubiak*,1(D), Rosalinda HGP Arends' ${ }^{1}$ Nancy Lee' ${ }^{1}$, Meina Liang ${ }^{2}$, Jianchun Zhang ${ }^{1}$ \\ \& Lorin K Roskos ${ }^{1}$ \\ ${ }^{1}$ Clinical Pharmacology and Safety Sciences, AstraZeneca PLC 1, One Medlmmune Way, Gaithersburg, MD 20878, USA \\ ${ }^{2}$ Clinical Pharmacology and Safety Sciences, AstraZeneca PLC 1, 121 Oyster Point Blvd, South San Francisco, CA 94080, USA \\ *Author for correspondence: Tel.: +1 301398 2770; Fax: +1 301398 7650; Robert.Kubiak@astrazeneca.com
}

\begin{abstract}
Aim: Competitive inhibition with excess unlabeled drug is used to confirm the presence of antidrug antibodies (ADA) in study samples. We evaluated specific and nonspecific responses from both drug-naive and drug-treated subjects to identify conditions required by the confirmatory assay to make accurate ADA classifications. Results: Nonspecific signal measured in drug-naive samples used to determine assay cut points was uniformly low and close to the screening cut point. Confirmatory assays performed on incurred study samples with nonspecific responses significantly above the level observed during cut point determination resulted in incorrect ADA classifications. Conclusion: Intensity of confirmatory response should be proportional to the screening response and therefore, to ensure accurate ADA classifications, the confirmatory responses cannot be considered as independent but need to be evaluated in relation to the screening responses.
\end{abstract}

First draft submitted: 24 November 2019; Accepted for publication: 28 January 2020; Published online: 24 February 2020

Keywords: antidrug antibodies $\bullet$ confirmatory cut point $\bullet$ immunogenicity $\bullet$ specific and nonspecific binding

Therapeutic proteins have an intrinsic potential to elicit an immune response in treated subjects and lead to generation of antidrug antibodies (ADA). Since ADA may significantly impact patient safety and drug efficacy, immunogenicity assays for detection of ADA are a critical component of any biotherapeutic drug development program $[1,2]$. The currently recommended strategy for testing of immunogenicity samples from clinical studies relies on tiered testing [3-5] where all subject samples are first tested for the presence of ADA in a high-throughput and sensitive screening assay. Samples with responses at or above the screening cut point are identified as potentially positive and tested in the second tier of analysis where the presence of ADA is confirmed in a specificity assay. This confirmatory or specificity tier is expected to reduce false positives generated in the screening assay $(\sim 5 \%)$ and ensure both high sensitivity and specificity of ADA detection [4].

A typical format employed for the confirmatory assay consists of competitive inhibition with excess of unlabeled drug. Specific signals caused by the presence of ADA should be suppressed by addition of the drug while the nonspecific signals should be minimally affected. It is not entirely clear what degree of signal change caused by addition of drug should be considered as indicative of a 'specific' versus a 'nonspecific' response. Several researchers proposed using statistical methods such as paired t-test where the signal drop caused by the addition of drug must meet a certain threshold of statistical significance [6,7]. These purely statistical approaches have been justly criticized for their failure to account for biological differences across the population of different subjects $[4,8]$.

The method currently recommended for determination of confirmatory cut point (CCP) is based on results from repeated analyses of at least 50 drug-naive samples from a relevant subject population tested in the presence and absence of excess drug. There is some controversy whether a CCP determined in drug-naive and, therefore by definition, ADA-negative samples, could be successfully applied to ADA-positive populations [9]. For this reason, some researchers prefer to spike drug-naive samples with a positive control antibody at a concentration corresponding to the assay's limit of detection and determine the CCP from responses obtained in the presence 
and absence of excess drug. While this approach provides some mimicry of incurred samples to be tested in the confirmatory tier, CCPs obtained in this fashion will depend on concentration, affinity and avidity of the positive control antibody selected for this exercise $[9,10]$.

We previously demonstrated that screening and confirmatory results obtained using competitive inhibition are correlated, which limits the ability of the confirmatory assay to generate results independent from the screening assay and reduce the percentage of false positives generated in the screening tier $[11,12]$. In this paper, we further elaborate on the relationship between screening and confirmatory results and show that low correlation between the screening and confirmatory results caused by a variable nonspecific signal will compromise the accuracy of classification in the confirmatory tier. The work presented demonstrates that the degree of signal change by addition of drug depends on the magnitude of the specific signal relative to the nonspecific signal. An increase of the nonspecific binding will lower the \%Inhibition of the specific signal and therefore, can lead to false confirmatory classifications since the CCP established during assay validation when nonspecific binding was lower, is no longer suitable for testing of incurred study samples.

\section{Experimental}

Reagents

Individual human serum samples and pooled human sera were obtained from BioIVT (NY, USA). Multiarray streptavidin-coated plates, Blocker A, Read Buffer T and ruthenium (II) sulfo-trisbipyridine N-hydroxysuccinimide ester were obtained from Meso Scale Discovery (MD, USA). EZ-Link biotin sulfo-N-hydroxysuccinimide ester was purchased from Thermo Scientific (IL, USA). Preparation of biotinylated and ruthenylated drug conjugates closely followed the procedures described elsewhere [13]. All data described here were obtained during clinical development of monoclonal antibody drug candidates 1 through 11 conducted by AstraZeneca (Table 1). Positive assay control was generated by AstraZeneca and consisted of affinity-purified monoclonal mouse antibody directed against the idiotype of antibody drug candidate 3 .

\section{Bioanalytical methods Immunogenicity assays}

All immunogenicity assays employed a solution-phase bridging format on Meso Scale Discovery platform following general procedures described previously [11,13]. Minimum required dilutions of samples and controls in assay buffer ranged from 10- to 100-fold. Final concentrations of antibody drugs used in the confirmatory assays ranged from 10 to $30 \mu \mathrm{g} / \mathrm{ml}$ and were sufficient to suppress the signal of high positive control samples (ranging from 1 to $10 \mu \mathrm{g} / \mathrm{ml}$ in neat matrix) to the assay background as recommended by the industry white papers $[4,10]$.

\section{Cut point determination}

Screening and confirmatory data for antibody biotherapeutic candidates 1 through 11 were obtained by testing $\geq 50$ drug-naive human serum samples from a relevant disease population. Each sample was tested multiple times by different analysts on different days with the exception of samples in immunogenicity assay B for ADA against Drug 10 (Table 1). To validate assay B, 305 drug-naive study samples were tested once. Responses in the presence and absence of drugs were normalized by dividing the raw signal by the mean signal of the negative control evaluated on each assay plate. Screening and CCPs were determined following the procedures described previously [14].

\section{Immunogenicity testing of study samples}

Clinical study samples for Drug 2 and Drug 10 were tested following a standard tiered approach. First, subject samples were tested in the screening assay and samples with a response higher than or equal to the screening cut point of 1.23 (Drug 2) or 1.32 (Drug 10) were considered as potentially ADA positive and tested in the confirmatory assay in the presence and absence of excess of respective drug. Samples with \%Inhibition $\geq 34.1 \%$ (Drug 2) or $28.0 \%$ (Drug 10) were classified as confirmed ADA positive. Immunogenicity testing for Drug 2 was suspended following a series of high nonspecific responses (Figure 5) and was resumed using a new preparation of ruthenylated Drug 2. Following unexpectedly high ADA-positive rates across all Drug 10 dose groups (Table 2), sample testing with the original assay (henceforth referred to as assay A) was stopped. New preparations of ruthenylated and biotinylated Drug 10 were generated using standard procedures; however, the final conjugated products were formulated in histidine-sucrose buffer as described previously [13]. The new immunogenicity assay (henceforth referred to as assay B) was re-validated using the newly prepared conjugated Drug 10 and the same analytical procedure as the 
Figure 1. Graphical representation of confirmatory assay using competitive inhibition with excess drug suppressing the specific signal in an antidrug antibodies positive sample. Blue and red bars indicate specific and nonspecific signal components, respectively. $S B(+D)$ : Normalized signal in the presence of drug; $S B(-D)$ : Normalized signal in the absence of drug.

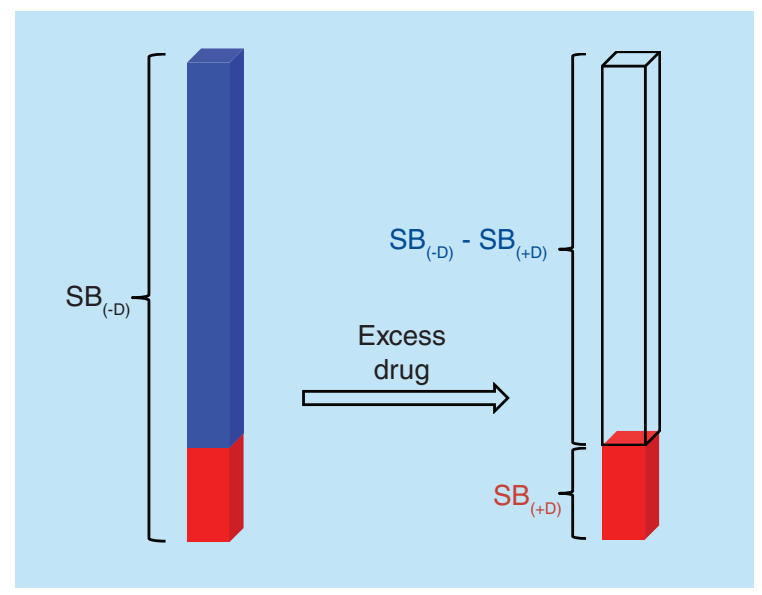

assay A. Retained immunogenicity ADA samples for Drug 10 were re-tested in assay B using screening (1.32) and confirmatory $(28.7 \%)$ cut points derived from 305 drug-naive study samples tested once.

\section{Results \& discussion}

Let us begin with an examination of the basic concepts underlying competitive inhibition of putative ADA signal with excess drug $[8,10]$. Signal change resulting from the addition of drug is typically expressed as \%Inhibition defined in Equation (1):

$$
\% \text { Inbibition }=\left(\frac{\operatorname{Signal}_{(-D)}-\text { Signal }_{(+D)}}{\operatorname{Signal}_{(-D)}}\right) 100 \%
$$

where Signal $_{(-D)}$ and Signal $(+D)$ represent raw signals in the absence and presence of drug, respectively. Alternatively, the raw signal can be normalized by dividing each response by the mean signal of the negative control (e.g., pooled serum) evaluated in each assay to obtain signal to baseline (SB) values. This operation allows comparing sample responses across different analytical runs with the assumption that the signals of the negative control and individual samples change in a similar fashion across different days, instruments and analysts [14]. The normalized expression is shown in Equation (2).

$$
\% \text { Inhibition }=\left(\frac{S B_{(-D)}-S B_{(+D)}}{S B_{(-D)}}\right) 100 \%
$$

Figure 1 provides a graphic representation of the confirmatory assay and helps understand the physical meaning of each term in Equation (2).

- $S B_{(+D)}$ - Normalized signal generated in the presence of drug (red in Figure 1). It is expected that addition of sufficient excess of drug should saturate all specific interactions in the sample and the remaining signal is due to factors other than ADA that do not interact with and therefore cannot be suppressed by the drug. These include, among others, nonspecific binding of conjugated reagents to the solid support or to matrix components such as rheumatoid factor, signal resulting from impurities in the reagents used to detect ADA (e.g., unreacted labeling agent, aggregated conjugated drug [13]), and background noise of the instrument.

- $S B_{(-D)}$ - Total normalized signal generated in the absence of drug. The total signal is composed of the nonspecific component $S B_{(+D)}$ defined above and of a specific component (blue in Figure 1). The specific signal is generated by matrix components (including but not limited to ADA) that specifically bind the drug used for capture and detection of ADA.

- It follows that the difference $S B_{(-D)}-S B_{(+D)}$ corresponds to the specific signal generated by the species that bind the drug. 


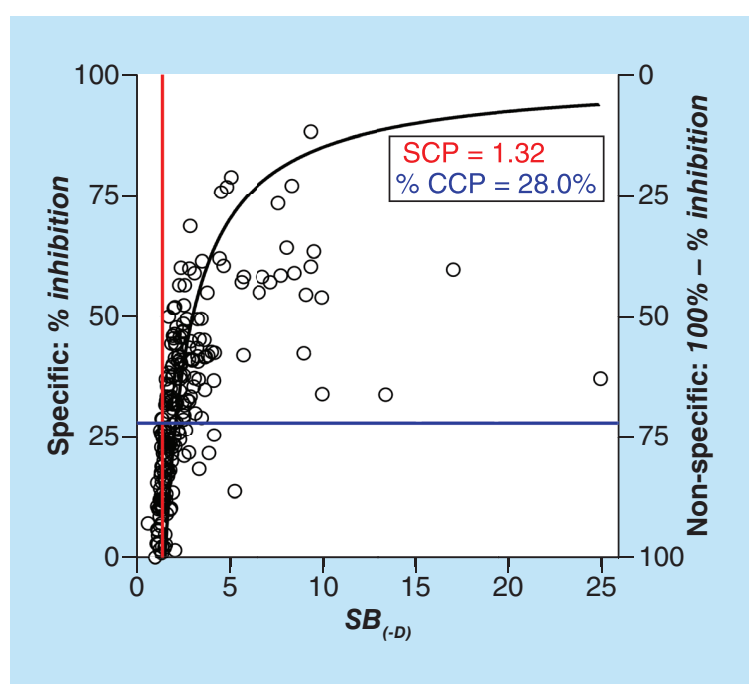

Figure 2. Screening and confirmatory responses for immunogenicity samples from Drug program 10 tested with assay A. Under the current paradigm, any response at or above both screening and CCPs is a confirmed ADA positive regardless of its non-specific component. CCP: Confirmatory cut point; SCP: Screening cut point.

As demonstrated above, \%Inhibition corresponds to the specific fraction of the total (i.e., screening) signal. By the same token, percentage of the nonspecific signal present in each sample is shown in Equation (3).

$$
\% \text { Nonspecific Signal }=100 \%-\% \text { Inhibition }=\frac{S B_{(+D)}}{S B_{(-D)}} 100 \%
$$

A close examination of Figure $1 \&$ Equations (1)-(3) points to significant problems underlying the current paradigm behind the use of competitive inhibition and fixed CCP for classification of ADA samples as discussed in the following sections.

Problem 1: \% Inhibition provides no information about the magnitude of the nonspecific response According to the current paradigm, any sample with \%Inhibition at or above the CCP is classified as positive for the presence of $\mathrm{ADA}$, regardless of the amount of nonspecific component in that sample. As shown in Equation (3), the nonspecific fraction in a confirmed ADA-positive sample can be as high as $100 \%$ - \%CCP. For example, if the CCP is $20 \%$, then a sample could be classified as ADA positive with as much as $80 \%$ of its signal originating from species that do not bind the drug. Such situations may be acceptable if the nonspecific signal consists mostly of the instrument background noise; however, high nonspecific signal originating from the sample itself will compromise the assay's ability to make accurate classifications. This is illustrated in Figure 2, which shows screening and confirmatory responses of samples from subjects treated with Drug 10 and tested with assay A. Multiple data points have similar \%Inhibition values and very different screening signals (and vice versa) due to differences in the amount of nonspecific signal. Additionally, nonspecific binding contributes more than half of the observed screening signal for many of the 'confirmed' ADA-positive samples. Such samples with relatively high screening responses and \%Inhibition values very close to the CCP may be misclassified as either true positives or as true negatives. In other words, \%Inhibition carries little information about the absolute amount of ADA in a sample, and therefore it is not reliable for making positive/negative classifications.

Problem 2: CCP is not valid if the nonspecific response is above the level observed during cut point determination

When screening and CCPs are determined, most samples from a drug-naive population show nonspecific responses $S B_{(+D)}$ below the screening cut point $(\sim 95 \%$ if the recommended false positive rate $[\mathrm{FPR}]=5 \%$ is used). This is because adding excess drug to a naive, and presumably ADA-negative sample, is not expected to result in an appreciable signal change. However, if the nonspecific responses $S B_{(+D)}$ observed during sample testing are much higher than those observed during cut point determination, there is no reason to expect that the screening and CCPs are still valid. According to Equation (3), for CCP to remain constant, an increase of $S B_{(+D)}$ should be accompanied by the same increase of $S B_{(-D)}$ and this assumption cannot be readily verified. Therefore, if the nonspecific $S B_{(+D)}$ responses observed during sample testing are much higher than screening cut point, then the CCP derived during assay validation is likely to result in misclassification of study samples. The resulting loss of the assay specificity may 
Figure 3. Specific (blue) and nonspecific (red) responses for samples confirmed as positive for the presence of antidrug antibodies against Drug 10 in assay $A$. The $\mathrm{X}$-axis shows the number of confirmed antidrug antibodies positive samples in the order of the screening response intensity.

$S B(+D)$ : Normalized signal in the presence of drug; $S B(-D)$ : Normalized signal in the absence of drug; SCP: Screening cut point.

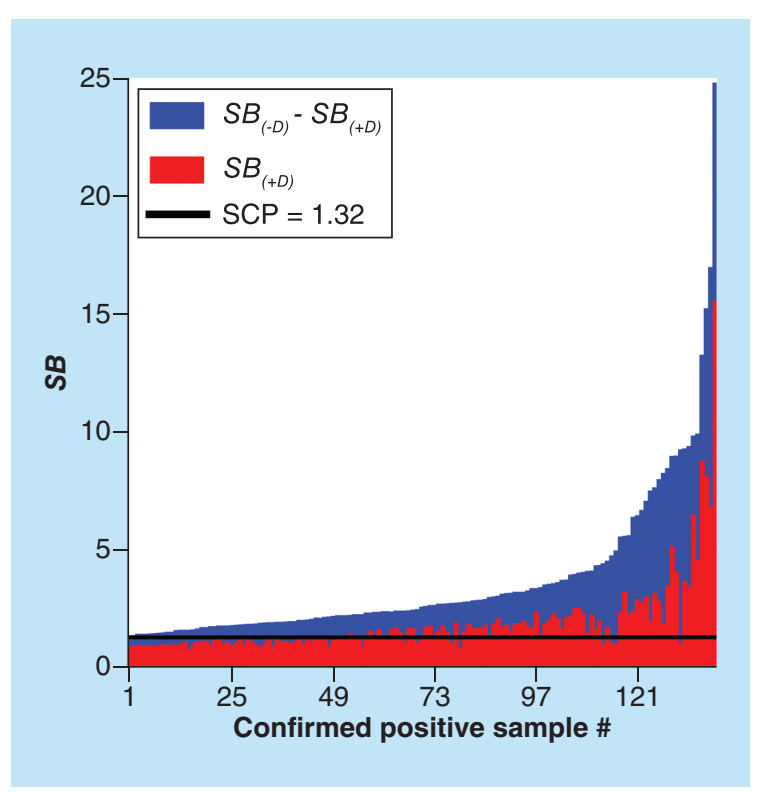

remain undetected without monitoring of $S B_{(+D)}$ responses during sample testing. This situation is illustrated in Figure 3, which shows the specific and nonspecific signal components for samples classified as confirmed positive for ADA against Drug 10 as measured with assay A. A large number of samples shown in this example have $S B_{(+D)}$ responses that are significantly higher than the screening cut point and, therefore, it is questionable whether the CCP originally derived from much smaller nonspecific signals is still able to make accurate positive/negative calls. Confirmed ADA-positive samples are typically titered down until their response reaches the screening or the titer cut point of the assay; however, for samples with very high $S B_{(+D)}$, the nonspecific signal is likely to make a significant contribution to the titer value as discussed below. It is doubtful whether such inaccurate ADA titers can be expected to show any correlation with the drug pharmacokinetics and pharmacodynamics observed in the study.

\section{Nonspecific signal in drug-naive populations}

Both problems with the current CCP paradigm indicate a special significance of the $S B_{(+D)}$ data, which while collected and used to calculate \% Inhibition, are typically not analyzed. We evaluated nonspecific responses acquired during determination of screening and CCPs for 11 different monoclonal antibody-drug candidates. For each drug program minimum, median, 99th percentile and maximum of the $S B_{(+D)}$ responses were determined. Immunogenicity assay for Drug 10 was initially validated using commercial drug-naive samples (assay A). Analytical method A was re-validated using naive study samples and conjugated Drug 10 stored in histidine-sucrose buffer (assay B). Results are summarized in Table 1.

\begin{tabular}{|c|c|c|c|c|c|c|c|c|c|c|c|c|}
\hline Drug program & 1 & 2 & 3 & 4 & 5 & 6 & 7 & 8 & 9 & $10^{A}$ & $10^{\mathrm{B}}$ & 11 \\
\hline Sample size (N) & 191 & 191 & 190 & 196 & 256 & 192 & 200 & 199 & 200 & 192 & 305 & 188 \\
\hline Minimum $S B_{(+D)}$ & 0.71 & 0.76 & 0.80 & 0.81 & 0.68 & 0.80 & 0.83 & 0.76 & 0.82 & 0.81 & 0.91 & 0.95 \\
\hline Median $S B_{(+D)}$ & 0.88 & 0.92 & 0.93 & 1.01 & 0.95 & 0.95 & 0.96 & 0.97 & 0.97 & 1.00 & 1.04 & 1.16 \\
\hline Maximum $S B_{(+D)}$ & 1.49 & 1.67 & 1.24 & 1.23 & 1.12 & 1.09 & 1.33 & 1.15 & 1.48 & 1.56 & 1.53 & 1.29 \\
\hline$S B_{(+D)}{ }^{99}$ & 1.43 & 1.36 & 1.18 & 1.22 & 1.10 & 1.07 & 1.26 & 1.12 & 1.40 & 1.45 & 1.35 & 1.27 \\
\hline SCP & 1.37 & 1.23 & 1.21 & 1.18 & 1.26 & 1.18 & 1.29 & 1.21 & 1.34 & 1.32 & 1.32 & 1.27 \\
\hline
\end{tabular}

Analytical method for program 10 was validated using commercial samples and ruthenylated/biotinylated Drug 10 formulated in phosphate-buffered saline (assay A) and re-validated using drug-naive study samples and conjugated Drug 10 formulated in histidine-sucrose buffer (assay B).

$S B(+D)$ : Normalized signal in the presence of drug; $S B\left(_{+D}\right)^{99}$ : 99th percentile of $S B_{(+D)}$ responses; $S C P$ : Screening cut point.

Addition of excess drug has little effect on responses of majority of samples in each population resulting in $S B_{(+D)}$ values close to 1 . Median $S B_{(+D)}$ values range from 0.88 to 1.16 indicating low and uniform nonspecific 
binding for all studied populations as well as optimal drug concentration used in each confirmatory assay, that is, high enough to suppress specific signal without affecting the nonspecific component. This observation is consistent with the maximum $S B_{(+D)}$ values being similar to or slightly higher than the final screening cut points calculated with $5 \%$ FPR.

Value $S B_{(+D)}^{99}$ corresponds to the 99th percentile of the nonspecific signal in each of the evaluated populations and could be considered as a conservative estimate of the nonspecific background observed after addition of excess drug. Intensity of the confirmatory response depends on the screening response and can be calculated as shown in Equation (4).

$$
\text { \%Inhibition } \geq\left(\frac{S B_{(-D)}-S B_{(+D)}^{99}}{S B_{(-D)}}\right) 100 \%
$$

By multiplying both sides of Equation (4) by $S B_{(-D)}$, one can transform it to a linear form where the specific signal is directly proportional to the screening signal.

$$
S B_{(-D)}-S B_{(+D)} \geq S B_{(-D)}-S B_{(+D)}^{99}
$$

Use of the larger than or equal to sign in Equations (4) and (5) signifies that $99 \%$ of the confirmatory responses observed in a given population should be higher than or equal to the value on the right side of the equation, that is, there is $1 \%$ chance of confirmatory response being lower than that predicted by the equation.

Figure 4 shows confirmatory responses measured for untreated subjects during validation of assay $10 \mathrm{~B}$ and fitted by Equation (4) using $S B_{(+D)}^{99}=1.35$ (Table 1). As explained above, $1 \%$ of confirmatory responses are below the fit line and the same can be expected from responses of ADA-positive subjects, that is, there is $1 \%$ chance that ADA-positive sample will give a confirmatory response lower than that predicted by Equation (4). Note that the use of 'classical' screening and CCPs results in about $2.3 \%$ of ADA positives in the untreated population rather than the nominal FPR of $1 \%$ or lower. One possible way to reduce the false-positive classifications in the untreated subjects is to set the screening cut point with $1 \%$ FPR rather than the recommended $5 \%$ FPR (i.e., increase it from 1.32 to $\sim 1.86$ ), and require confirmatory responses of samples with $S B_{(-D)} \geq 1.86$ to be equal to or higher than those calculated by Equation (4). In the proposed approach, an ADA-positive sample should measure at or above the screening cut point calculated with $1 \%$ FPR and its confirmatory response should agree with that calculated by Equations (4) or (5). This approach not only reduces the number of false-positive classifications but is expected to maintain it at the level set during assay validation.

The ability of Equations (4) and (5) to predict confirmatory responses of ADA-positive samples was evaluated for the positive control antibody used in drug program 3. Pooled serum was spiked with a mouse monoclonal antibody against Drug 3 at concentrations ranging from $6.4 \mathrm{ng} / \mathrm{ml}$ to $210 \mu \mathrm{g} / \mathrm{ml}$ and tested in the confirmatory assay. Results presented in Figure 5 show a satisfactory prediction of confirmatory responses across three orders of magnitude of the screening signal suggesting that simple equations such as (4) and (5) could be successfully applied to describe very high confirmatory responses that may be observed in ADA-positive subjects.

\section{Monitoring of nonspecific responses in drug-treated populations}

It is understandable that reagents used for detection of ADA may display a certain level of nonspecific binding to matrix or assay components. While complete elimination of the nonspecific binding by manipulating the labeling ratios or the reagent concentrations may be difficult, it is crucial to maintain it at a constant and low level over the course of sample testing. A significant increase of the nonspecific signal may require re-validation of the assay to avoid inaccurate sample classifications and therefore, monitoring of the nonspecific signal during sample analyses may help detect problematic assay performance. Figure 6 shows chronological $S B_{(+D)}$ values measured during testing of immunogenicity samples from a clinical study of Drug 2. Samples 1 through 394 were tested with the original lots of ruthenylated and biotinylated Drug 2, and most of the observed $S B_{(+D)}$ responses were below the critical value determined during assay validation, that is, $S B_{(+D)}^{99}=1.36$ (Table 1). Samples 395 through 458 were tested with a new batch of ruthenylated Drug 2. This was associated with a cluster (42 out of 64 values) of $S B_{(+D)}$ responses higher than $S B_{(+D)}^{99}$. Testing was paused, and the elevated nonspecific signal was traced back to the presence of high molecular aggregates in the new preparation of ruthenylated Drug 2 . Another batch of ruthenylated reagent was prepared and sample testing was resumed including re-screening of samples 395-458. 
Figure 4. Screening and \%Inhibition responses for untreated subjects used to determine screening and confirmatory cut points for assay $10 \mathrm{~B}$. Red and green vertical lines show screening cut points calculated using false positive rate $=5 \%$ and $1 \%$, respectively. Solid black line corresponds to Equation (4) with $S B_{(+D)}^{99}=1.35$. Under the new approach, confirmed positive responses fall between the green and the black lines rather than simply above the blue (CCP) line.

CCP: Confirmatory cut point; SCP: Screening cut point.
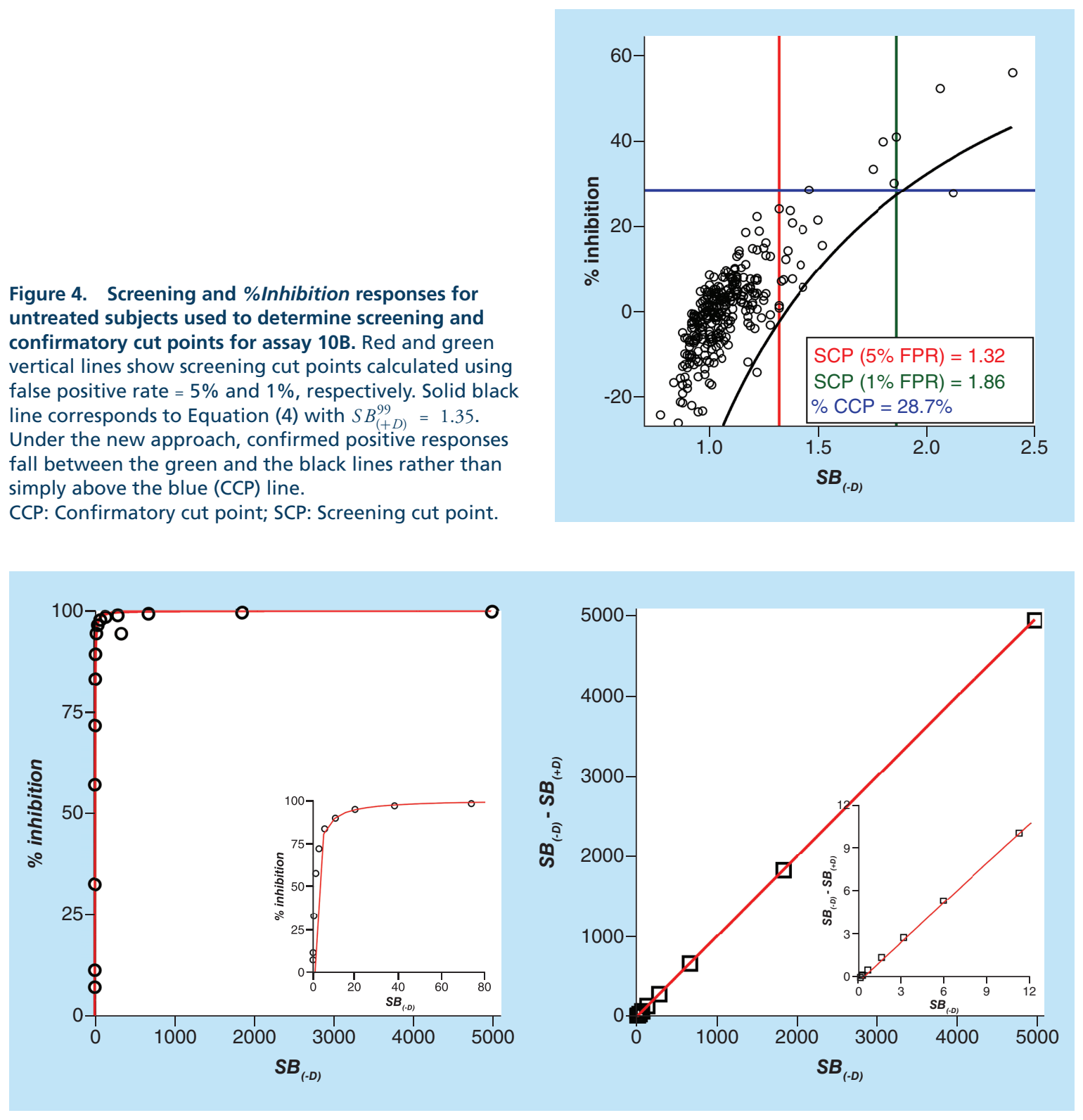

Figure 5. Screening and confirmatory responses of the positive control antibody used in drug program 3. Observed confirmatory responses are overlaid with the responses predicted by Equation (4) (left panel) and Equation (5) (right panel) respectively, with $S B_{(+D)}^{99}=1.18$. The insets show confirmatory results for $S B_{(-D)}$ less than 80 .

$S B(+D)$ : Normalized signal in the presence of drug; $S B(-D)$ : Normalized signal in the absence of drug.

Nonspecific signal obtained with the new batch of ruthenylated Drug 2 (samples 459-1166) measured below 1.36 for most of the tested samples indicating that the assay returned to the originally validated performance.

It is worth noting that in this particular case, in-study monitoring of nonspecific responses enabled detecting a problem with ruthenylated drug more efficiently than a 'traditional' reagent qualification performed using assay controls and commercial drug-naive samples.

\section{Impact of high nonspecific signal on accuracy of classification}

Providing that sufficient excess of drug is used in the confirmatory assay, there is no reason to believe that Equation (4) could not be used to describe confirmatory responses for samples containing treatment-induced ADA. However, most \%Inhibition values observed in subjects treated with Drug 10 and tested in assay A (Figure 2) do not follow the function described by Equation (4) with many 'confirmed positive' samples showing a very high nonspecific signal 


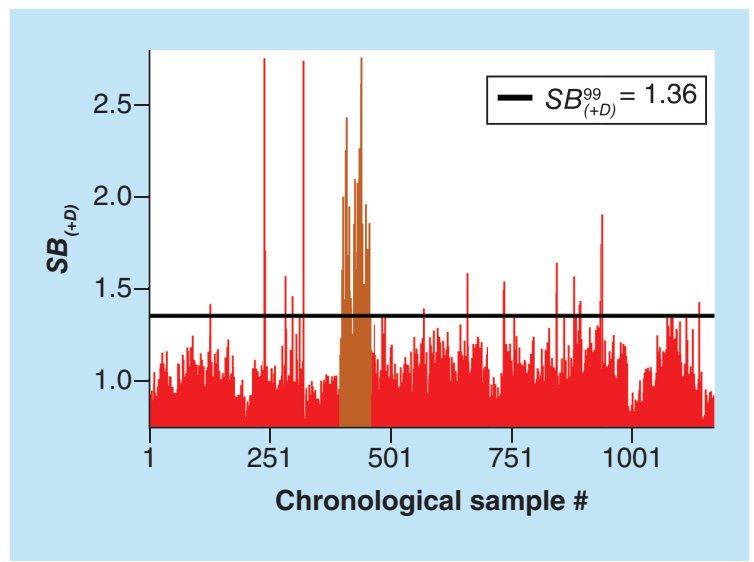

Figure 6. Chronological $S B_{(+D)}$ values measured in a confirmatory assay for the presence of antidrug antibodies in samples from subjects treated with Drug 2. Cluster of high responses with $S B_{(+D)}^{99}>1.36$ marked in orange (samples 395-458) was associated with using a batch of ruthenylated Drug 2 containing high molecular aggregates.

$S B(+D)$ : Normalized signal in the presence of drug.

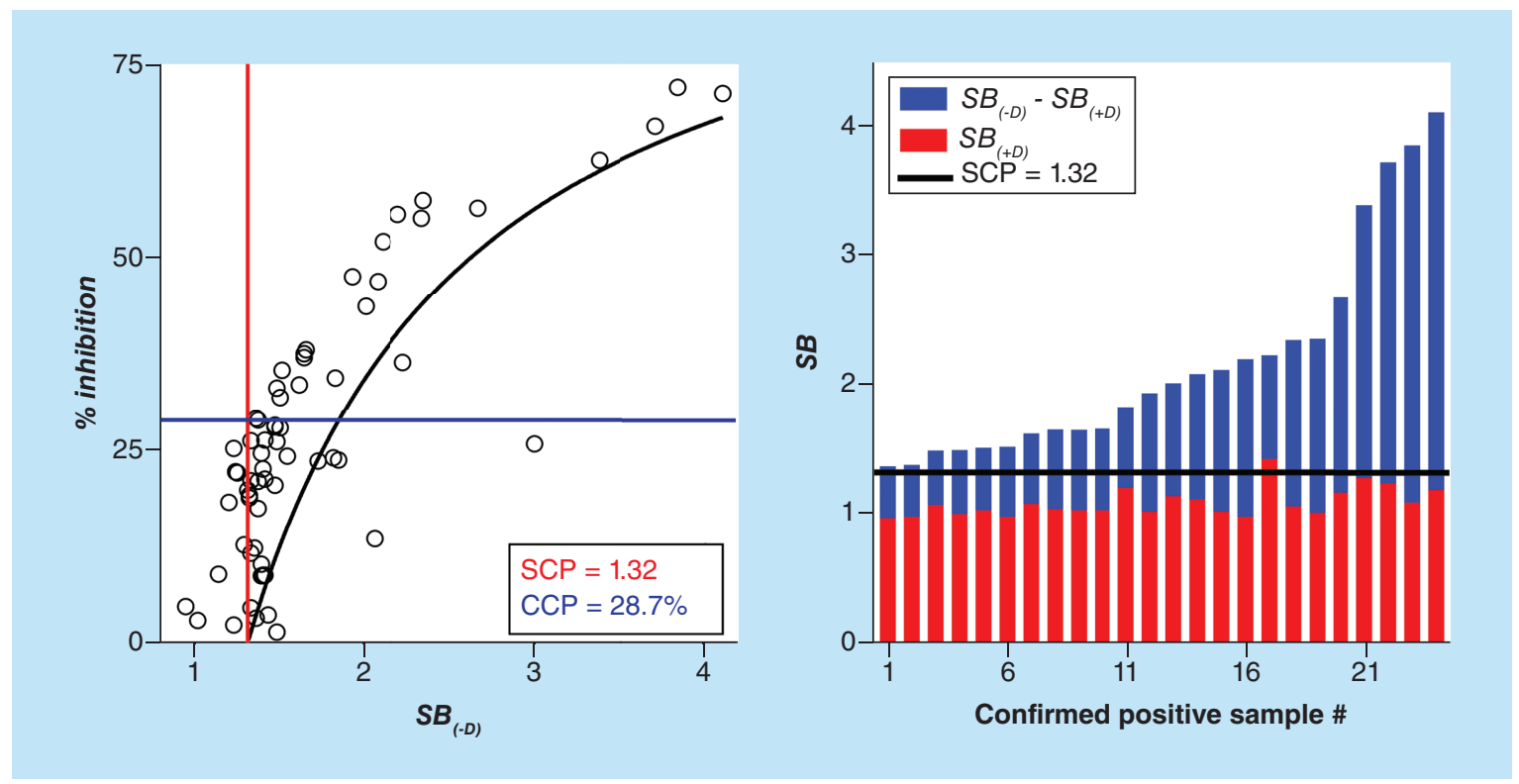

Figure 7. Specific and non-specific responses for immunogenicity samples from Drug program 10 tested with assay B. Left panel: samples with \% Inhibition responses below the fit line calculated with Equation (4) using $S B_{(+D)}^{99}=1.35$ display non-specific responses higher than $99 \%$ of those observed during determination of SCPs and CCPs. Right panel: specific (blue) and non-specific (red) responses for samples confirmed as positive for the presence of ADA against Drug 10. The $\mathrm{X}$-axis shows the number of confirmed ADA positive samples in the order of the screening response intensity.

CCP: Confirmatory cut point; $S B(+D)$ : Normalized signal in the presence of drug; $S B(-D)$ : Normalized signal in the absence of drug; SCP: Screening cut point.

(Figure 3). It is unlikely that insufficient amount of drug was used in the confirmatory assay since the screening responses observed in the assay A $\left(S B_{(-D)}<25\right)$ were well within the responses that could be completely suppressed as shown in Figure 5. As discussed above, the unexpected increase of the nonspecific signal suggested that the assay departed from the original validated performance, which could be caused by degradation of conjugated reagents [13].

New preparations of ruthenylated and biotinylated Drug 10 were generated and formulated in histidine-sucrose buffer, which was previously shown to prevent formation of high molecular aggregates [13]. New screening and CCPs were derived from responses measured in drug-naive samples from subjects enrolled in the clinical study (predose and placebo) and were found to be similar to the original cut points obtained during prestudy validation with commercial samples. All study samples were re-tested using validated assay B and confirmatory responses are presented in Figure 7. Contrary to the original results (Figures $2 \& 3$ ), the nonspecific signals were low and uniform across the confirmed positive samples. 
A dramatic reduction of intensity and frequency of the nonspecific responses was accompanied by a similar decrease in the number of ADA-positive samples as shown in Table 2. ADA incidence and prevalence for Drug 10 measured with assay A ranged from 17.0 to $23.3 \%$ across all dose groups including placebo, while $\mathrm{ADA}$ incidence/prevalence measured with assay $\mathrm{B}$ was much lower with $\mathrm{ADA}$ rates ranging from 2.9 to $3.9 \%$. Median/maximum titer values measured with assays A and B were 120/7680 and 90/960, respectively. This provides a clear illustration of how the presence of high nonspecific component in the screening signal can confound not only classification in the confirmatory assay but also titer measurement as discussed above. The significant reduction of ADA rates and titers upon retesting with new conjugated reagents indicates problematic assay performance rather than elevated nonspecific signal in the study samples caused by the presence of species such as rheumatoid factor or immunoglobulins $\mathrm{M}$. It is worth pointing out that both assays A and B were fully validated and results shown in Table 2 are reported from analytical runs that met acceptance criteria. Screening and CCPs determined for both the assays were almost identical suggesting that there were no significant differences between the drug-naive commercial and study samples used for cut point determination. Sensitivity and drug tolerance measured with assays A and B were very similar and sufficient for the study needs (data not shown) and therefore, the lower ADA rates observed with assay B could not be explained by a substandard assay performance.

It may be tempting to simply dismiss the results generated by assay A as caused by some problem with the critical reagents, but this explanation does not address the fact that confirmatory tier designed to eliminate false-positive classifications essentially failed to do so. According to the current paradigm, both assays A and B generated valid immunogenicity results, however, this work argues that the current paradigm needs to be revised to include a requirement for uniformly low nonspecific signal. Only samples tested in assay B displayed low and consistent nonspecific signal and therefore only the results obtained with assay B should be considered as valid. This is illustrated in Figure 7, where confirmatory responses show a good agreement with those predicted by Equation (4) using $S B_{(+D)}^{99}=1.35$, a value derived from drug-naive subjects during validation of assay B (Table 1). This observation suggests that ADA classification in the confirmatory assay could be based on Equation (4) rather than on a fixed CCP. In this alternative approach, to be classified as ADA positive, a sample would have to give a screening response that is at or above the screening cut point and confirmatory response sufficiently close to the predicted $\%$ Inhibition value.

\begin{tabular}{|c|c|c|}
\hline \multirow[t]{2}{*}{ Dose group } & \multicolumn{2}{|c|}{ ADA incidence/prevalence } \\
\hline & Samples tested with assay A & Samples tested with assay B \\
\hline Placebo & $17.6 \%$ & $3.9 \%$ \\
\hline Dose group I & $17.0 \%$ & $3.0 \%$ \\
\hline Dose group II & $23.3 \%$ & $2.9 \%$ \\
\hline
\end{tabular}

\section{Conclusion}

We examined the assumptions underlying the use of CCP in tiered immunogenicity testing. We argue that \%Inhibition by itself is insufficient to make positive/negative classifications without taking into account the specific and nonspecific signal components. Accurate confirmatory classifications require the nonspecific component $S B_{(+D)}$ to be similar to the levels observed during cut point determination in samples from drug-naive subjects. As long as the $S B_{(+D)}$ values remain below a certain critical threshold established during assay validation (e.g., 99th percentile of the population or $S B_{(+D)}^{99}$ ), confirmatory response can be accurately predicted from the screening response using Equations (4) or (5). This consideration points to a conclusion that confirmatory response is related to the screening response and therefore, CCP is not essential for making ADA classifications. Confirmatory assay could be used to check for consistency of assay performance by monitoring if the nonspecific signal does not exceed $S B_{(+D)}^{99}$ or a similar 'nonspecific threshold'. If during sample testing, the frequency and magnitude of the nonspecific responses are significantly higher than those observed during cut point determination, the assay most likely departed from the original validated performance and therefore it can no longer generate accurate screening or confirmatory classifications.

Interdependence of the screening and confirmatory assays discussed in this and other publications $[11,12,15]$ presents a significant obstacle for the current tiered approach. If the confirmatory tier is unable to make classifications 
that are independent from the screening tier, false positives generated in the screening assay may not be effectively removed by the confirmatory assay. This calls for decreasing of the FPRs used for screening cut point determination from $5 \%$ down to $1 \%$ or lower to avoid contaminating immunogenicity results with too many random false-positive classifications [16]. For nonorthogonal immunogenicity assays such as those discussed here, the 'traditional' ADA classification relying on a sequential application of screening and CCPs could be replaced by fitting the observed \%Inhibition to a simple mathematical function relating screening and confirmatory responses such as that proposed in Equations (4) and (5). In this approach, classification in the confirmatory assay would not be based on a fixed CCP but rather on the degree of agreement between the observed \%Inhibition with that predicted by Equations (4) or (5).

\section{Future perspective}

While a decrease of signal upon addition of drug is undeniable evidence for the presence of species that bind the drug in a specific manner, establishment of a CCP as a fixed \%Inhibition value that can separate the specific responses from the nonspecific ones may be very difficult if not impossible. At the same time, measuring of nonspecific responses can be used to monitor integrity of critical reagents during sample testing.

While all examples presented in this work were generated using a solution-phase bridging format on electrochemiluminescence platform, our analyses are general and can be applied to any assay where competitive inhibition with drug (or its fragments) is used to make claims about the ADA status of test samples. Evaluating study data as presented in this work can help ensure that the assay performs as validated and retains its ability to generate accurate classifications. Additionally, equations and graphs shown here can be used during assay development to ensure low and uniform nonspecific signal for drug-naive samples and to determine the concentration of drug in the confirmatory assay. Plotting the specific signal versus the screening signal using Equation (5) is especially useful since, if the assay performs adequately, it should give a straight line with a slope close to 1 across all screening responses. From this then follows that the specific signal should be directly proportional to the amount of ADA present in the sample and therefore, measuring the $S B_{(-D)}-S B_{(+D)}$ values could potentially provide a semiquantitative characterization of $\mathrm{ADA}$ and replace the titer assay [17].

This work demonstrates that intensity of the confirmatory response depends on the screening response. Rather than using a CCP, ADA classifications should be based on a mathematical function relating screening and confirmatory responses. It also could be argued that competitive inhibition with excess drug is not a good method for making positive/negative classifications but instead, is better suited for semiquantitative characterization of ADA, which could replace a titer value. Alternatively, the current testing strategy may still be valid; however, criteria for ADA assays should be modified and more factors should be considered when developing fit-for-purpose confirmatory assays. Such an approach would necessitate a significant revision of the current immunogenicity testing paradigm and the present work is a first step in this direction.

\section{Financial \& competing interests disclosure}

The authors have no relevant affiliations or financial involvement with any organization or entity with a financial interest in or financial conflict with the subject matter or materials discussed in the manuscript. This includes employment, consultancies, honoraria, stock ownership or options, expert testimony, grants or patents received or pending, or royalties.

No writing assistance was utilized in the production of this manuscript.

\section{Ethical conduct of research}

The authors state that they have obtained appropriate institutional review board approval or have followed the principles outlined in the Declaration of Helsinki for all human or animal experimental investigations. In addition, for investigations involving human subjects, informed consent has been obtained from the participants involved.

\section{Open access}

This work is licensed under the Attribution-NonCommercial-NoDerivatives 4.0 Unported License. To view a copy of this license, visit http://creativecommons.org/licenses/by-nc-nd/4.0/

\section{References}

Papers of special note have been highlighted as: $\bullet$ of interest; $\bullet \bullet$ of considerable interest 
Summary points

\section{Experimental}

- Analyses of results from immunogenicity assays for detection of antidrug antibodies (ADA) were performed to define conditions required to make accurate classifications in the confirmatory tier. Data were generated by solution-phase bridging assays on electrochemiluminescence platform performed on samples from drug-naive and treated subjects from 11 different drug-development programs.

\section{Results \& discussion}

- Confirmatory response can be broken down to two components; nonspecific (signal in the presence of excess drug) and specific (the difference between the screening signal and the nonspecific signal). Ideally, the nonspecific response should remain constant and at the level observed during determination of cut points in drug-naive individuals.

- In-study monitoring of the nonspecific responses can be useful for detecting problems with integrity of critical reagents. Maintaining the nonspecific responses below the screening cut point can help ensure that the assay generates accurate classifications and titers that properly reflect the amount of ADA in each sample.

\section{Conclusion}

- A confirmational cut point expressed as \% Inhibition is insufficient to make accurate positive/negative classifications without considering the magnitude of the specific and nonspecific signal components.

- A valid confirmatory response should be proportional to the screening response and therefore, a confirmational cut point is not essential for making ADA classifications.

1. Guidance for industry. Immunogenicity assessment for therapeutic protein products (2014). www.fda.gov/downloads/drugs/guidances/ucm338856.pdf

2. European Medicines Agency. Guideline on immunogenicity assessment of therapeutic proteins. London,UK (2017). www.ema.europa.eu/docs/enGB/document library/Scientific_guideline/2017/06/WC500228861.pdf

3. Koren E, Smith HW, Shores E et al. Recommendations on risk-based strategies for detection and characterization of antibodies against biotechnology products. J. Immunol. Methods 333, 1-9 (2008).

4. Shankar G, Devanarayan V, Amaravadi L et al. Recommendations for the validation of immunoassays used for detection of host antibodies against biotechnology products. J. Pharm. Biomed. Anal. 48(5), 1267-1281 (2008).

5. US FDA. Guidance for industry. Immunogenicity testing of therapeutic protein products - developing and validating assays for anti-drug antibody detection. Rockville, MD, USA (2019). www.fda.gov/media/119788/download

6. Neyer L, Hiller J, Gish $\mathrm{K}$ et al. Confirming human antibody responses to a therapeutic monoclonal antibody using a statistical approach. J. Immunol. Methods 315, 80-87 (2006).

7. Jaki T, Allacher P, Horling F. A false sense of security? Can tiered approach be trusted to accurately classify immunogenicity samples? J. Pharm. Biomed. Anal. 128, 166-173 (2016).

-• Demonstrates that \%Inhibition cannot be trusted to make accurate antidrug antibody classification.

8. Mikulskis A, Yeung D, Chen W et al. Novel data analysis methods to overcome cut point challenges and enable comprehensive assessment of antidrug binding activity in confirmatory assays. J. Immunol. Methods 392, 38-48 (2013).

9. Smith HW, Moxness M, Marsden R. Summary of confirmation cut point discussions. AAPS J. 13(2), 227-229 (2011).

10. Jani D, Marsden R, Mikulskis A et al. Recommendations for the development and validation of confirmatory anti-drug antibody assays. Bioanalysis 7(13), 1619-1631 (2015).

-• White paper summarizing development and validation of confirmatory assays.

11. Kubiak RJ, Zhang L, Zhang J et al. Correlation of screening and confirmatory results in tiered immunogenicity testing by solution-phase bridging assays. J. Pharm. Biomed. Anal. 74, 235-245 (2013a).

- Presents arguments in a discussion on correlation of screening and confirmatory results.

12. Kubiak RJ. Regarding confirmatory immunogenicity assays: response by the authors. J. Pharm. Biomed. Anal. 84, 175-176 (2013b).

- Presents arguments in a discussion on correlation of screening and confirmatory results.

13. Kubiak RJ, Lee N, Zhu Y et al. Storage conditions of conjugated reagents can impact results of immunogenicity assays. J. Immunol. Res. 1485615 (2016). http://dx.doi.org/10.1155/2016/1485615

14. Zhang L, Zhang JJ, Kubiak RJ, Yang H. Statistical methods and tool for cut point analysis in immunogenicity assays. J. Immunol. Methods 389, 79-87 (2013).

15. Coleman D, Hendricks R, Delarosa R et al. Regarding confirmation of immunogenicity assays. J. Pharm. Biomed. Anal. 84, 173-175 (2013).

- Presents arguments in a discussion on correlation of screening and confirmatory results. 
16. Kubiak RJ, Zhang J, Ren P et al. Excessive outlier removal may result in cut points that are not suitable for immunogenicity assessments. J. Immunol. Methods 463, 105-111 (2018).

- Summarizes discussions on the best way to determine a CCP.

17. Starcevic Manning M, Kroenke MA, Lee SA et al. Assay signal as an alternative to titer for assessment of magnitude of an antidrug antibody response. Bioanalysis 9(23), 1849-1858 (2017). 